\title{
VALVULAR DISEASE
}

\section{TAVI noninferior to surgery in intermediate-risk patients}

extension
of TAVI to
intermediate-
risk patients
had to be
expected

Transcatheter aortic valve implantation (TAVI) with a self-expanding prosthesis is noninferior to surgery in patients with severe aortic stenosis and who are at intermediate surgical risk. This finding, from the randomized SURTAVI trial, was presented at the ACC.17 Scientific Sessions and published in NEJM.

TAVI is superior to medical therapy in inoperable high-risk patients, and might be preferable to surgery in patients who are at high surgical risk. However, data on the efficacy and safety of TAVI in patients at intermediate surgical risk were limited. Accordingly, the SURTAVI investigators recruited 1,746 patients with severe, symptomatic aortic stenosis and who were at intermediate surgical risk (Society of Thoracic Surgeons Predicted Risk of Mortality $4.5 \pm 1.6 \%$ ). The trial was funded by Medtronic.

Overall, 864 patients were randomly assigned to undergo TAVI, and 796 patients were allocated to surgical aortic valve replacement. Of the patients assigned to TAVI, $84 \%$ received a first-generation CoreValve bioprosthesis, and $16 \%$ received a second-generation Evolut R device.

Outcomes were assessed using a Bayesian analysis when 1,400 patients had reached 12 months of follow-up. In this analysis, the incidence of the primary end point (a composite of all-cause death or disabling stroke at 24 months) was lower in the TAVI group (12.6\%) than with surgery (14.0\%), meaning that the criteria for noninferiority of TAVI compared with surgery were met.

Compared with TAVI, surgery was associated with a higher rate of atrial fibrillation ( $43.4 \%$ versus $12.9 \%$ ), acute kidney injury ( $4.4 \%$ versus $1.7 \%$ ), requirement

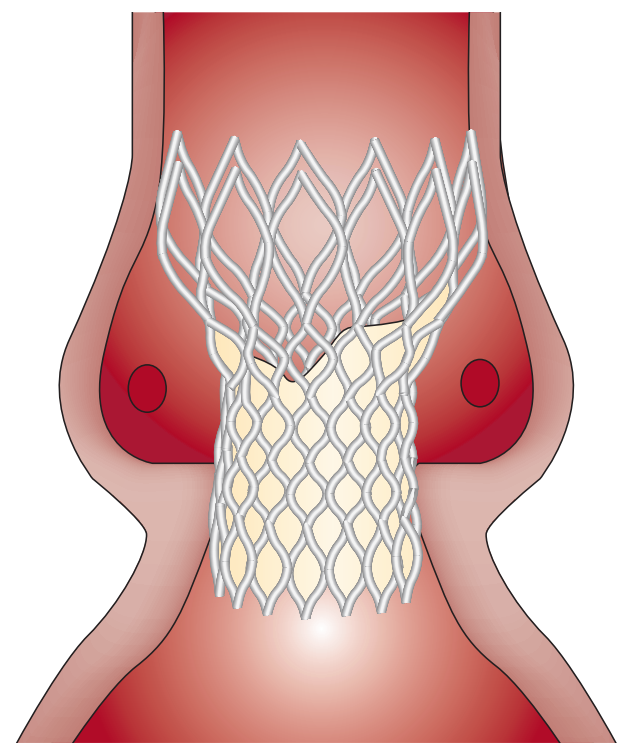

Reproduced from http://www.nature.com/nrcardio/journal/v9/n8/ $\underline{\text { full/nrcardio.2012.58.html }}$ for $>4$ units of blood transfusion ( $12.7 \%$ versus $3.6 \%$ ), and stroke at 30 days ( $5.6 \%$ versus $3.4 \%$ ). Conversely, TAVI was associated with a higher rate of permanent implantation of a pacemaker (25.9\% versus $6.6 \%$ ); however, mortality was not increased in patients who required a new pacemaker.

Aortic valve haemodynamics improved in both groups. Compared with the surgical group, patients who underwent TAVI had larger aortic valve areas and lower mean aortic valve gradients. However, moderate or severe residual paravalvular regurgitation was higher with TAVI than with surgery ( $5.3 \%$ versus $0.6 \%$ ).

Michael Reardon, lead author of the trial report, notes that even with $84 \%$ use of first-generation prosthetic valves (which, unlike second-generation devices, cannot be recaptured, repositioned, or redeployed), TAVI still performed favourably compared with excellent surgical outcomes. These findings "should lead to approval of this valve for intermediate-risk patients and are likely to move intermediate-risk TAVI to a class I indication the next time the guidelines are revised," says Reardon.

Given the rapid worldwide uptake of TAVI in inoperable or high-risk patients, "extension of TAVI to intermediate-risk patients had to be expected," comments Professor Ottavio Alfieri from the San Raffaele Scientific Institute, Milan, Italy, and who was not involved in the SURTAVI trial. However, "in the context of intermediate-risk patients (with a longer life expectancy after the procedure compared with inoperable or high-risk patients), the durability of the TAVI devices is an important issue. In my opinion, due to the unknown durability of TAVI devices, patients aged $<75$ years at intermediate surgical risk should still be treated surgically." Furthermore, Alfieri believes that intermediate-risk patients "should be allocated to TAVI only when the anatomy is favourable, and the procedure can be expected to be performed safely and be effective (without significant residual aortic regurgitation)". Assessment will be the responsibility of the Heart Team, who should evaluate the size of the aortic annulus, location of the coronary arteries, presence of heavy calcifications, and other features.

Michael Reardon and colleagues are now enrolling patients into a randomized trial to assess the use of TAVI in patients at low surgical risk.

Gregory B. Lim

ORIGINAL ARTICLE Reardon, M. J. et al. Surgical or transcatheter aortic-valve replacement in intermediate-risk patients. N. Engl.J. Med.http://dx.doi.org/10.1056/NEIMoa1700456 (2017) FURTHER READING Arsalan, M. \& Walther, T. Durability of prostheses for transcatheter aortic valve implantation. Nat. Rev. Cardiol. 13, 360-367 (2016)| Taramasso, M. et al. New devices for TAVI: technologies and initial clinical experiences. Nat. Rev. Cardiol. 11, 157-167 (2014) 